\title{
Relaciones interpersonales y desempeño laboral en hoteles turisticos del departamento de Puno
}

\section{Interpersonal relations and work performance in tourist hotels of the department of Puno}

\author{
Miriam Serezade Hancco Gomez a 0000-0003-3913-2775 \\ Amira Carpio Maraza ${ }^{\mathrm{b}}$ 0000-0002-1917-4938 \\ Zoraima Julieta Laura Castillo ${ }^{\mathrm{c}}$ 0000-0001-7460-6740
}

Universidad Nacional del Altiplano, Puno, Perú.

a mhancco@unap.edu.pe
${ }^{\mathrm{b}} \underline{\text { acarpio@unap.edu.pe }}$
${ }^{\mathrm{z}}$ zjlaura@unap.edu.pe

Recibido: 23/06/2021

Aprobado: 25/08/2021

Publicado: 15/09/2021

\section{Resumen}

Las relaciones interpersonales constituyen un papel crítico en una empresa debido a la interacción con personas que conservan puntos de vista, ideas, percepciones o formación diferentes, las mismas que pueden afectar para bien o mal en el desempeño laboral, por ende, permitir o distraer el logro de los objetivos organizacionales. En ese contexto la investigación tiene como objetivos: analizar cómo son las relaciones interpersonales de los colaboradores de los hoteles del departamento de Puno y determinar el desempeño laboral de los colaboradores de los hoteles del departamento de Puno. Los métodos enmarcan una investigación de enfoque cuantitativo, de alcance exploratoriodescriptivo y diseño no experimental. La población 186 está conformada por 40 hoteles categorizados en tres estrellas, de la misma se ha obtenido una muestra de 16 hoteles, que albergan 84 colaboradores a ser encuestados. La técnica de recolección de datos fue la encuesta y el instrumento el cuestionario para la variable relaciones interpersonales, que contiene 15 preguntas medidas a una escala de Likert y para la variable desempeño laboral se aplicó la evaluación por el método de escala gráfica. El proceso de datos se ha dado a través de la hoja de cálculo Excel y el paquete estadístico SPSS. Mediante este se calculó las frecuencias y porcentajes de cada variable y sus dimensiones. Los resultados permiten concluir que las relaciones interpersonales en los hoteles del departamento de Puno según escala del instrumento utilizado son buenas, así el desempeño laboral de los colaboradores de los hoteles del departamento de Puno es bueno según la escala grafica.

Palabras clave: colaboradores; desempeño laboral; organizaciones; relaciones interpersonales.

\begin{abstract}
Interpersonal relationships constitute a critical role in a company due to the interaction with people who hold different points of view, ideas, perceptions or training, which can affect job performance for good or bad, therefore, allowing or distracting the achievement of organizational objectives. In this context, the research aims: to analyze the interpersonal relationships of the employees of the hotels in the Puno department and to determine the work performance of the employees of the hotels in the Puno department. The methods frame a research with a quantitative approach, exploratorydescriptive scope and non-experimental design. The population is made up of 40 hotels categorized in three stars, from which a sample of 16 hotels has been obtained, which house 84 collaborators to be surveyed. The data collection technique was the survey and the instrument was the questionnaire for the interpersonal relationships variable, which contains 15 questions measured on a Likert scale and for the job performance variable, the evaluation was applied by the graphic scale method. The data processing has been done through the Excel spreadsheet and the SPSS statistical package. Through this, the frequencies and percentages of each variable and its dimensions were calculated. The results allow to conclude that the interpersonal relationships in the hotels of the Puno department according to the scale of the instrument used are good, thus the work performance of the employees of the hotels of the Puno department is good according to the graphic scale.
\end{abstract}

Keywords: collaborators; job performance; organizations; interpersonal relationships. 


\section{Introducción}

Las relaciones interpersonales son aquellas que se establecen entre al menos dos personas y son parte esencial de la vida en sociedad. Esto significa que, en todo espacio sociocultural, un individuo convive con otros individuos, lo que le permite conocer a los demás y conocerse a sí mismo (Moreno y Perez, 2018). Las empresas son escenarios propicios para ello. Éstas hoy en día, se encuentran en una constante búsqueda de propuestas de valor, Del Toro (2015), son conscientes de la imagen que se forma el cliente, favorable o desagradable de la calidad del producto o servicio que está recibiendo, Diaz et al. (2014) así se vuelve imperante definir estrategias, donde el comportamiento de su personal es fundamental para el logro de sus objetivos.

Díaz et al. (2014) en ese contexto las relaciones interpersonales constituyen un papel crítico en una empresa debido a la interacción con personas que conservan puntos de vista, ideas, metas, percepciones o formación diferentes, Torres y Zegarra (2015) las mismas que deben alcanzar objetivos. Arias et al. (2018), donde una fuente de dificultades es la falta de acuerdos claros y explícitos. Por ello Tamayo (1997), Chiavenato (2007) y Robbins y Coulter (2013), proponen su estudio haciendo énfasis en dimensiones como la comunicación, el trato y las actitudes, considerándolas como esenciales para el desempeño laboral. A lo anterior complementa Yañez et al. (2010), la calidad de las relaciones interpersonales en sí no basta para mejorar el desempeño laboral, pero sí puede contribuir significativamente a él, para bien o para mal.

Díaz et al. (2014) la evaluación del desempeño laboral tiene importantes ventajas tanto para las organizaciones como para los colaboradores. Una de sus principales aportaciones es la de aumentar el conocimiento de la organización sobre cómo se está realizando el trabajo. Robbins y Coulter (2013) por lo tanto determina qué tan exitoso ha sido un individuo en el logro de sus actividades y objetivos. Complementa Indacochea et al. (2018) y Chiavenato (2007) todo trabajador debe poseer un conjunto de competencias básicas para desarrollar sus actividades. Robbins (1999) la medición de resultados se limita a formular la pregunta ¿hiciste el trabajo?; Boada (2019) algunos métodos de evaluación son la autoevaluación, evaluación de $90^{\circ}$, evaluación de $180^{\circ}$, comités de evaluación y evaluación de $360^{\circ}$. Chiavenato (2000) pero la evaluación del desempeño es una apreciación sistemática del desempeño de cada persona, puede llevarse a cabo tomando como dimensiones: conocimiento del cargo, calidad del trabajo, responsabilidad e iniciativa.

En investigaciones realizadas, La Peña (2012), Vásquez (2015), Bejarano (2019) y Salazar (2018) afirman que las relaciones interpersonales positivas propician un buen ambiente laboral, complementan a estos Muñoz (2017) y Santos (2016) señalando que un nivel regular de relaciones interpersonales representa una oportunidad de mejorar el desempeño laboral. Para Morillo y Cardozo (2017), el sector turismo no escapa a la realidad de la globalización, ni a los retos actuales que tiene la empresa.

El sector hotelero viene emprendiendo esfuerzos, es consciente que para alcanzar objetivos requiere, escenarios enlosquesedebeestablecercomunicación constante entre sus colaboradores, generando así relaciones interpersonales que deberían ser sanas, sin embargo, suele surgir muchas discrepancias, recelos, roces etc.; aspectos aun no considerados; generalmente se relacionaba que un mal servicio al cliente estaba limitado a inadecuadas prácticas de áreas como marketing, operaciones, hasta finanzas; mas no de aspectos entre colaboradores, siendo las relaciones interpersonales pieza fundamental para el desempeño laboral. En ese contexto el propósito de la investigación es dar una mirada integral y describir el comportamiento de estas dos variables, para ello se ha establecido como objetivos: i) analizar cómo son las relaciones interpersonales de los colaboradores de los hoteles del departamento de Puno y ii) determinar el desempeño laboral de los colaboradores de los hoteles del departamento de Puno.

\section{Marco teórico}

\section{Relaciones interpersonales}

Una relación interpersonal es una interacción recíproca entre dos o más personas (Díaz et al., 2014). Las relaciones interpersonales como un aspecto básico en la vida del hombre, funcionando no sólo como un medio para alcanzar determinados objetivos sino un fin en sí mismo. El hombre por naturaleza es un ser social que necesita relacionarse y no puede estar aislado de otras personas (Cornejo y Tapia, 2011). 
Desde el punto de vista empresarial, las relaciones interpersonales se entienden como la capacidad que tiene la persona de cooperar y trabajar con sus compañeros, estableciendo una meta a conseguir y organizando el trabajo diario para no entorpecer el desempeño profesional de otros. Todas las personas establecen numerosas relaciones a lo largo de su vida, con sus padres, amistades o compañeros de trabajo y estudio. A través de ellas intercambian formas de sentir y de ver la vida; también comparten necesidades, intereses y afectos.

Se puede cultivar las buenas relaciones humanas con las demás personas, teniendo con ello una comunicación efectiva, y practicando la interacción, además de tomar en cuenta que cada persona es diferente a otra y dependiendo de las características de cada una se definirá una conducta buena o mala de nosotros hacia ellos, las relaciones se van mejorando a medida que se va conociendo al individuo brindándole un trato prudente y respetuoso.

\section{Dimensiones de las relaciones interpersonales}

La comunicación interpersonal, es el proceso por el cual los colaboradores en una organización intercambian sentimientos e información mediante mensajes verbales y no verbales. Constituye un elemento fundamental en las relaciones humanas (Díaz et al., 2014). Ignorar la comunicación produce errores, fallos, retrasos, esperas, disculpas, ${ }_{18}$ pretextos, problemas o conflictos con compañeros del grupo de trabajo, con jefes, subordinados y con personal de otros departamentos. Cuando la comunicación y sus formas no suceden dentro de un marco regulado y formal que la contenga, entonces las relaciones informales ganan espacio peligrosamente para la organización, generando un ambiente negativo.

Las actitudes de los colaboradores, es definida como la disposición interna aprendida y duradera que sostiene las respuestas favorables o desfavorables del individuo dentro de una organización (Yañez et $a l ., 2010)$. Existe una gran variedad de conflictos que se originan a raíz de malas actitudes, donde los principales conflictos son: latente, cuando el conflicto es aquel que existe, pero no se manifiesta claramente y manifiesto, cuando el conflicto se manifiesta de forma patente.
También, la confianza del trabajador es la seguridad emocional que lo lleva a pensar que la empresa es responsable y se preocupa por él (San Martín, 2013). Lo anterior mencionado por los autores, inducen a pensar que los elementos de la cultura organizacional influyen en el comportamiento de los colaboradores y por consiguiente en su desempeño. Asimismo las creencias y los significados compartidos, refuerzan el compromiso y a partir de ello pueden tener efectos positivos en el esfuerzo individual y colectivo (Alvarado y Monroy, 2013).

Los tratos por parte de los jefes, el buen trato laboral no son sólo las conductas concretas con que las personas se relacionan, sino que también, es el conjunto de normas, costumbres, prácticas y valores que existen en relación con esto (Cornejo y Tapia, 2011). El comportamiento que tienen los colaboradores en la empresa contribuye a que se trabaje en armonía, logre un excelente clima laboral, el personal incremente su productividad y la empresa logre los objetivos planeados. De la misma manera que las emociones y las creencias de las personas impulsan su comportamiento y sus elecciones, las personas también crean conexiones emocionales con su entorno laboral, con su trabajo, con los clientes y proveedores, con lo que usan y consumen en su vida cotidiana.

Una capacidad de gobernabilidad hace referencia a las habilidades aprendidas y ejercidas por la alta gerencia de una organización para establecer una estructura de gobierno y unas condiciones de gobernabilidad que le permitan orientar, administrar e integrar las actividades de las unidades operativas, monitorear su desempeño y tomar las decisiones sobre asignación de recursos que aseguren la supervivencia de la organización (Dávila, 2013). Así mismo, uno de los aspectos que interviene en el desempeño laboral de los colaboradores es el estilo de liderazgo del jefe (Willman y Velasco, 2011).

\section{Desempeño laboral}

El desempeño es una actuación orientada a un resultado, es decir, algo observable, medible y dinámico, puesto que el desempeño es acción, es una secuencia de acontecimientos conductuales perceptibles producidos por una persona (Willman y Velasco, 2011). Por su para Guisao et al. (2017) señala que el desempeño organizacional se compone de desempeño financiero y desempeño 
no financiero, donde el desempeño no financiero, alude al aumento de la satisfacción del cliente, el mejoramiento de la imagen corporativa, el aumento del valor de las marcas y de la productividad de los colaboradores.

Asimismo, el desempeño laboral son aquellas acciones o comportamientos de los colaboradores en la búsqueda de los objetivos relevantes para la organización y que pueden ser medidas en términos de las competencias de cada individuo y sus niveles de contribución a la empresa (Chiavenato, 2007). Es probable que el desempeño sea alto cuando se han fijado metas motivadoras, están presentes los moderadores y funcionan los mediadores. Si bien una capacidad se define en referencia a un nivel de desempeño, este varía en cada organización de manera que se evita (Dávila, 2013). Cuando una empresa lleva a cabo una evaluación del rendimiento de sus colaboradores persigue objetivos tanto administrativos como motivacionales, buscando, en última instancia, una mejora de sus resultados organizativos (Serrano y Barba, 2012).

La administración del desempeño laboral es el proceso mediante el cual la empresa asegura que el colaborador trabaje alineado con las metas de la organización, así como las practicas a través de las cuales el trabajo es definido y revisado, las capacidades son desarrolladas y las recompensas son distribuidas en las organizaciones. Cada corriente pretende, desde su perspectiva, resolver cuestiones que conciernen a un mismo tema: el del desarrollo de las competencias laborales como una alternativa para lograr mejorar la productividad del trabajo y los resultados de la organización.

Se considera más conveniente adoptar un enfoque integrado, donde se mezclen los elementos de las tres corrientes que resultan ventajas, y que facilitan la implantación del enfoque de competencias en la gestión del capital humano (Sánchez et al., 2017). Teniendo en cuenta el análisis anterior, los elementos que caracterizarían un enfoque integrado para el estudio de las competencias son los siguientes: Atención en las características de los colaboradores que más rinden o con un desempeño excelente, utilización del análisis funcional como metodología central para la determinación de competencias y la Inclusión de todos los niveles de la organización en la determinación de competencias.

\section{Dimensiones del desempeño laboral}

El conocimiento del cargo es la comprensión de un conjunto de responsabilidades específicas para un trabajo, así como la capacidad permanente para mantenerse al tanto de cambios en funciones de trabajo (Vargas y Flores, 2019), se considera el dominio y familiarización con las actividades del cargo que desempeña los colaboradores. Así mismo, la calidad del trabajo, es importante para la evaluación de desempeño laboral, pues son los resultados derivados de la participación de los colaboradores en el lugar de trabajo (Chiavenato, 2007), se considera la capacidad minuciosa, pulcritud y dedicación que pone el colaborador en sus labores.

Otra dimensión es la responsabilidad, donde se ve el cumplimiento a las obligaciones y ser cuidadoso al tomar decisiones o al realizar algo. En esta dimensión se considera la actualización del colaborador para solucionar en forma oportuna situaciones difíciles. También, la iniciativa es otra dimensión, que es considerada como el concepto activo de rendimiento y recoge la idea de que los colaboradores en el trabajo pueden realizar tanto las tareas asignadas como desarrollar sus propias metas, e incluso iniciar por sí mismo otras (Sanín y Salanova, 2014), se considera la habilidad del colaborar para integrarse con otros y ejecutar un trabajo.

El trabajo en equipo es considerando como el impacto más significativo de un equipo está en el hecho de alcanzar más cosas unidos que de manera individual. Otorga empoderamiento a cada uno de los miembros, eliminando los obstáculos que pueden impedirles hacer sus tareas correctamente. Promueve estructuras de trabajo más flexibles y con menos jerarquía (Indacochea et al., 2018), cuando los colaboradores se reúnen y satisfacen un conjunto de necesidades se produce una estructura que posee un sistema estable de interacciones dando origen al equipo de trabajo. Esta dimensión considera la habilidad del colaborador para integrarse con otros y ejecutar un trabajo.

\section{Metodología}

La investigación se enmarca en un enfoque cuantitativo, de alcance exploratorio - descriptivo; describe, analiza y predice los fenómenos que ocurre en los hoteles categorizados como tres estrellas del departamento de Puno, aplicando 
la lógica deductiva. Valiéndose del análisis de cuadros estadísticos y el análisis documental (Hernández et al., 2010). El diseño es no experimental, se observó los fenómenos y la realidad de las relaciones interpersonales y el desempeño personal, tal como se dan en su contexto natural de situaciones ya existentes que no fueron provocadas intencionalmente. La población está comprendida por 40 hoteles categorizados en tres estrellas adecuados al DS.11-2019-MINCETUR, para el análisis y tratamiento de información el muestreo es no probabilístico por conveniencia, debido al acceso a la información. Del total de la población la muestra está conformada por 16 hoteles turísticos, de los cuales se ha considerado 84 colaboradores a ser encuestados, los mismos que se desenvuelven en diferentes puestos según sus estructuras orgánicas.

Para el logro de los objetivos, se analizó las relaciones interpersonales, para ello se utilizó como técnica de recolección de datos, la encuesta y como instrumento el cuestionario, conformado por 15 preguntas que contemplan las tres dimensiones: comunicación, actitudes y trato; medidos a una escala de Likert donde $1=$ deficiente, 2 = malas, $3=$ regulares, $4=$ buenas y $5=$ muy buenas. Para evaluar el nivel de desempeño laboral se aplicó la evaluación de desempeño laboral por el método de escala gráfica propuesta por Chiavenato (2002), la misma que sirve para calificar en forma más objetiva posibles las dimensiones respecto al aspecto laboral y personal de los colaboradores, encaminados a tipificar su rendimiento, donde los factores son conocimiento del cargo, calidad de trabajo, responsabilidad, trabajo en equipo, iniciativa y actitud y sus respectivos grado son $\mathrm{E}$ de 0 a1, D de 5 a $8, \mathrm{C}$ de 9 a 12, B de 13 a 16 y A de 17 a 20 , después de esta evaluación se traslada los resultado a la escala de análisis e interpretación del nivel de desempeño, donde la escala es insuficiente de 0 a 40, deficiente de 41 a 58, regular de 59 a 74, bueno de 75 a 88 y óptimo de 89 a 100.

Una vez aplicada la encuesta y la evaluación del desempeño a los 84 colaboradores se desarrolló el procedimiento de análisis de datos, que se da a través de la hoja de cálculo Excel y el paquete estadístico SPSS. Mediante este se calcula las frecuencias y porcentajes de cada variable y sus respectivas dimensiones. Para dar soporte a la interpretación de los resultados se utilizó la técnica de recolección de datos observación, la cual se tomó en visitas anónimas. El periodo de estudio comprende los meses de marzo a octubre del 2019.

\section{Resultados y discusión}

Para analizar las relaciones interpersonales, se utilizó las dimensiones: comunicación, actitudes y trato. En la tabla 1 se observa como son las relaciones interpersonales en los hoteles turísticos de acuerdo con cada dimensión.

Tabla 1. Dimensiones de las relaciones interpersonales en los hoteles de la ciudad de Puno.

\begin{tabular}{lllllll}
\hline Dimensiones & \multicolumn{2}{c}{ Comunicación } & \multicolumn{2}{c}{$\begin{array}{l}\text { Actitudes de los } \\
\text { colaboradores }\end{array}$} & \multicolumn{2}{c}{$\begin{array}{l}\text { Trato hacia los } \\
\text { colaboradores }\end{array}$} \\
\hline Escala & Fi & \% & Fi & \% & Fi & \% \\
\hline Deficientes & 6 & $7.1 \%$ & 5 & $5.9 \%$ & 8 & $9.5 \%$ \\
Malas & 11 & $13.1 \%$ & 12 & $14.3 \%$ & 10 & $11.9 \%$ \\
Regulares & 17 & $20.2 \%$ & 15 & $17.9 \%$ & 15 & $17.9 \%$ \\
Buenas & 37 & $44.1 \%$ & 28 & $33.3 \%$ & 38 & $45.2 \%$ \\
Muy buenas & 13 & $15.5 \%$ & 24 & $28.6 \%$ & 13 & $15.5 \%$ \\
\hline TOTAL & $\mathbf{8 4}$ & $\mathbf{1 0 0} \%$ & $\mathbf{8 4}$ & $\mathbf{1 0 0 \%}$ & $\mathbf{8 4}$ & $\mathbf{1 0 0 \%}$ \\
\hline
\end{tabular}

En la tabla 1, se observa que respecto a la dimensión comunicación dentro de los hoteles turísticos, el $44.1 \%$ mencionaron que la comunicación dentro de su empresa es buena, un $20.2 \%$ menciona que es regular, seguido de porcentajes menores. Para corroborar este resultado la investigación de Vásquez (2015), aplicado a 50 colaboradores, identificó que la comunicación y el trato son los factores que tienen presencia en la empresa, existiendo confianza, comprensión, cariño $\mathrm{y}$ afecto.

Asimismo, la investigación de Moreno y Perez (2018) aplicado a 62 colaboradores, estableció que al mantener buenas relaciones interpersonales incrementa el nivel productivo. Este escenario es discordante con los hallazgos de Parra (2012) que identificó que muchas empresas no tienen flujos 
de comunicación, prima la jerarquía por ende no se logran los objetivos. Así mismo, Muñoz (2017) concluye que la comunicación en la empresa es regular con tendencia a mala.

Respecto a la actitud que tiene un colaborador respecto a su trabajo se observa que el $33.3 \%$ mencionaron que las actitudes son buenas, al interactúa con personas, emite conductas a través de las actitudes, las cuales son percibidas de manera positiva, negativa o neutra por las personas que nos rodean. También el 5.9\% mencionaron que las actitudes son deficientes y un $14.3 \%$ son malas. Este resultado coincide con la investigación de Muñoz (2017) donde el 39.7\% manifiestan tener malas actitudes. Asimismo, en la investigación de Diaz et al. (2014) y Martinez (2004) identificaron que el $48 \%$ de los colaboradores están comprometidos con la empresa.

Respecto al trato que reciben por parte de sus jefes, el $45.2 \%$ señalaron que el trato es bueno, un $17.9 \%$ mencionan que son regulares, la relación jefe - subordinado es cordial. En la investigación de Muñoz (2017) realizadas el $27.9 \%$ sus colaboradores manifiestan un nivel malo y el $45.6 \%$ un nivel regular, siendo la causa primordial la presión por lograr los objetivos.
Tabla 2. Relaciones interpersonales en los hoteles de la ciudad de Puno.

\begin{tabular}{lll}
\hline Escala & Fi & $\mathbf{\%}$ \\
\hline Deficientes & 6 & $7.1 \%$ \\
Malas & 11 & $13.2 \%$ \\
Regulares & 16 & $19.0 \%$ \\
Buenas & 34 & $40.5 \%$ \\
Muy buenas & 17 & $20.2 \%$ \\
TOTAL & $\mathbf{8 4}$ & $\mathbf{1 0 0 \%}$ \\
\hline
\end{tabular}

De acuerdo a la tabla 2, podemos mencionar que las relaciones interpersonales en los hoteles turísticos son buenas para un $40.5 \%$ de los colaboradores y un $19.0 \%$ consideran que son regulares.

Para corroborar esta información en la investigación de Arias et al. (2018) identifico que las relaciones interpersonales están más ligadas a los aspectos emocionales de los colaboradores en un $42.8 \%$. Por su lado La Peña (2012) identifico que el 38\% de los colaboradores confunde las relaciones interpersonales con la simpatía, compenetración o afinidad.

Para evaluar el nivel de desempeño laboral, se consideró el modelo de Chiavenato (2004) basado en cinco indicadores: conocimiento del cargo, calidad de trabajo, responsabilidad, trabajo en equipo e iniciativa (tabla 3 ).

Tabla 3. Evaluación por parte de los jefes o administradores del desempeño laboral de los colaboradores.

\begin{tabular}{|c|c|c|c|c|c|c|c|c|c|c|}
\hline \multirow[t]{3}{*}{ GRADOS } & \multicolumn{10}{|c|}{ FACTORES DE VALORACIÓN } \\
\hline & \multicolumn{2}{|c|}{$\begin{array}{l}\text { Conocimiento } \\
\text { del cargo }\end{array}$} & \multicolumn{2}{|c|}{$\begin{array}{l}\text { Calidad de } \\
\text { trabajo }\end{array}$} & \multicolumn{2}{|c|}{ Responsabilidad } & \multicolumn{2}{|c|}{ Iniciativa } & \multicolumn{2}{|c|}{$\begin{array}{l}\text { Trabajo } \\
\text { en equipo }\end{array}$} \\
\hline & $\mathbf{F i}$ & $\%$ & $\mathbf{F i}$ & $\%$ & Fi & $\%$ & $\mathbf{F i}$ & $\%$ & Fi & $\%$ \\
\hline E (4) & 7 & $8.3 \%$ & 4 & $4.8 \%$ & 8 & $9.5 \%$ & 5 & $0 \%$ & 1 & $1.2 \%$ \\
\hline D (8) & 22 & $26.2 \%$ & 24 & $28.6 \%$ & 22 & $26.2 \%$ & 20 & $23.8 \%$ & 22 & $26.2 \%$ \\
\hline C (12) & 8 & $9.5 \%$ & 7 & $8.3 \%$ & 6 & $7.1 \%$ & 9 & $10.7 \%$ & 10 & $11.9 \%$ \\
\hline B (16) & 35 & $41.7 \%$ & 32 & $38.1 \%$ & 33 & $39.3 \%$ & 36 & $42.9 \%$ & 34 & $40.5 \%$ \\
\hline A (20) & 12 & $14.3 \%$ & 17 & $20.2 \%$ & 15 & $17.9 \%$ & 14 & $16.7 \%$ & 17 & $20.2 \%$ \\
\hline TOTAL & 84 & $100 \%$ & 84 & $100 \%$ & 84 & $100 \%$ & 84 & $100 \%$ & 84 & $100 \%$ \\
\hline
\end{tabular}

En la tabla 3, se observa cómo es la calificación por parte de los dueños o administradores de los hoteles turísticos, se observa que respecto al conocimiento del cargo el $41.7 \%$ de los colaboradores obtuvieron una calificación B, sin embargo existe un $26.2 \%$ con una calificación $\mathrm{D}$, respecto a la dimensión calidad de trabajo el $38.1 \%$ de los colaboradores obtuvieron una calificación de $\mathrm{B}$ y un $28.6 \%$ una calificación $\mathrm{B}$, respecto a la responsabilidad el $39.3 \%$ obtuvieron una calificación $\mathrm{B}$ y un $26.2 \%$ D.
Respecto a dimensión iniciativa el $42.9 \%$ de los colaboradores obtuvieron una calificación B y un $23.8 \%$ D. Finalmente respecto a la dimensión trabajo en equipo el $40.5 \%$ obtuvieron una calificación B y un $26.2 \%$ D. denotando así la existencia de una proporción mejor al 50\% que tienen una calificación mala o deficiente.

Teniendo en cuenta la tabla 3 de la calificación del desempeño laboral por parte de los dueños a administradores de los hoteles, se obtuvo la tabla 4 
donde se observa el nivel de desempeño laboral de la totalidad de los colaboradores.

Tabla 4. Desempeño laboral según escala de valoración en los hoteles de la Ciudad de Puno.

\begin{tabular}{lll}
\hline VALORES CUALITATIVOS & Fi & $\mathbf{\%}$ \\
\hline Optimo & 15 & $17.9 \%$ \\
Bueno & 34 & $40.5 \%$ \\
Regular & 8 & $9.5 \%$ \\
Deficiente & 22 & $26.2 \%$ \\
Insuficiente & 5 & $5.9 \%$ \\
TOTAL & $\mathbf{8 4}$ & $\mathbf{1 0 0 \%}$ \\
\hline
\end{tabular}

El $40.5 \%$ de los colaboradores tienen un desempeño laboral bueno y el $17.9 \%$ óptimo, denotando que los colaboradores conocen sus funciones y responsabilidades, tienen iniciativa para solucionar problemas y tomar decisiones, hace que el desempeño laboral sea bueno. Asimismo la investigación de Vásquez (2015) aplicada a 50 colaboradores, identifico que el $46 \%$ presenta un desempeño laboral. También la investigación de Chiang y San Martin (2015) identifico que el desempeño laboral es regular.

\section{Conclusiones}

Según los resultados obtenidos las relaciones interpersonales en los hoteles del departamento de Puno son buenas, pues las dimensiones: comunicación, actitudes y trato se practican de forma frecuente, que se manifiestan por adecuados canales y sistemas de comunicación, en donde se evidencia que la información se transmite de forma clara y eficiente, los colaboradores manifiestan que tienen buena actitud frente a su trabajo y manifiestan que existe un trato adecuado por parte de sus jefes.

Respecto al desempeño laboral de los colaboradores de los hoteles del departamento de Puno se identificó que es bueno, pues sus dimensiones revelan que se practican de manera frecuente, los colaboradores se encuentran satisfechos con su trabajo, son responsables con el cumplimiento de sus tareas, disfrutan trabajar en equipo y tienen iniciativa para resolver los conflictos que se presenten.

\section{Conflictos de interés}

Los autores declaran no tener ningún tipo de conflicto de intereses.

\section{Agradecimiento}

Nuestro agradecimiento a la Universidad Nacional del Altiplano por el apoyo en la facilitación de la biblioteca y otros servicios. Agradecemos a los colaboradores de los hoteles turísticos del departamento de Puno por su apoyo y participación en la investigación. Así mismo, a la alta dirección y empresarios hoteleros por permitirnos realizar la investigación en sus hoteles.

\section{Referencias bibliográficas}

Alvarado, Ó. y Monroy, R. (2013). Cultura organizacional en una empresa propiedad de sus trabajadores. Cuadernos de Administracion, 26(47), 259-283. Retrieved from https://revistas.javeriana.edu.co/index. php/cuadernos admon/article/view/7111

Arias, W., Lazo, J. y Quintana, S. (2018). ¿Es el clima organizacional determinante de relaciones interpersonales o son las relaciones interpersonales las que determinan el clima organizacional? Revista Industrial Data, 21(2), 81-90. 10.15381/idata.v21i2.15606

Bejarano, M. (2019). Relaciones interpersonales afectivas y desempeño laboral según variables sociodemográficas de los trabajadores de radio y televisión de Lima Metropolitana. Revista de Investigación En Psicología, 22(2), 313328. 10.15381/rinvp.v22i2.17431 ISSN

Boada, N. (2019). Satisfacción laboral y su relación con el desempeño laboral en una Pyme de servicios de seguridad en el Perú. Journal of Economics, Finance and International Business, 3 (October), 75-103. 10.20511/ jefib.2019.v3n1.398

Chiang, M. y San Martin, J. (2015). Análisis de la Satisfacción y el Desempeño Laboral en los Funcionarios de la Municipalidad de Talcahuano. Ciencia \& Trabajo de La Universidad Del Bio Bio, 17(54), 159-165. Retrieved from www.cienciaytrabajo.cl

Chiavenato, I. (2000). Administración de Recursos humanos (Mc Graw Hi). Colombia.

Chiavenato, I. (2002). Gestión del talento humano (Mc Graw-Hi). Mexico.

Chiavenato, I. (2007). Administración de recursos humanos: El capital humano de las organizaciones (Mc Graw-Hi). Mexico.

Cornejo, M. y Tapia, M. L. (2011). Redes sociales $\mathrm{y}$ relaciones interpersonales en internet. Fundamentos En Humanidades, XII(24), 219-229. Retrieved from http://www.redalyc. 
org/articulo.oa? id $=18426920010 \% 0 \mathrm{~A}$

Dávila, J. C. (2013). Capacidades organizacionales: Dinámicas por naturaleza. Cuadernos de Administración, 26(47), 11-33. Retrieved from https://www.researchgate.net/ publication/313489543 Capacidades Organizacionales Dinamicas por naturaleza

Del Toro, A. (2015). Gestión de las relaciones interpersonales para lograr el éxito en la empresas de servicios. Revista Interamericana de Ambiente y Turismo, 11(1), 75-83. $\underline{10.4067 / 305}$

Díaz, D., Hernández, E., Isla, R., Delgado, N., Luis, D., y Rosales, C. (2014). Factores relevantes para aumentar la precisión, la viabilidad y el éxito de los sistemas de evaluación del desempeño laboral. Papeles Del Psicólogo, 35(2), 115-121. Retrieved from http://www. redalyc.org/articulo.oa?id $=77831095004$

Diaz, M. G., Peña, M. y Castellanos, B. (2014). El liderazgo y las Relaciones interpersonales dentro del clima organizacional. Revista Global de Negocios, 2(1), 1-10. Retrieved from http://ssrn.com/abstract=2324967

Guisao, S., Rincón, L. D. y Arias, J. (2017). Capacidad de tecnologías de información y desempeño organizacional: efecto mediador de la capacidad de absorción. Cuadernos de Administracion, 30(55), 37-65. 10.11144/ Javeriana.cao30-55.ctido

Hernández, R.; Fernández, R. y Baptista, P. (2010). Metodología de la Investigación (Mc GRAW Hi). México.

Indacochea, B., Porraspita, D. y Indacochea, B. (2018). El desempeño laboral: un problema socialdelaciencia.Revistade La Construcción, IX(2), 45-62. Recuperado de https://dialnet. unirioja.es/servlet/articulo?codig

La Peña, Y. (2012). Estudio fenomenológico de las relaciones interpersonales laborales: la mirada del personal de enfermería de las diferentes unidades del Hospital General Santa Bárbara de Soria (Tesis doctoral) (Universidad de Alicante). Retrieved from www.wltallerdigital.com

Martinez, R. (2004). Las relaciones interpersonales en la Empresa. Realidad Empresarial, (3), 37-38. 10.5377/reuca.v0i3.5999

Moreno, S. y Perez, E. (2018). Relaciones interpersonales en el clima laboral de la universidad tecnológica del Chocó Diego. Revista CES Derecho, 9(1), 13-33. 10.21615/ cesder.9.1.2

Morillo, M. y Cardozo, C. D. C. (2017). Sistema de costos basado en actividades en hoteles cuatro estrellas del Estado Mérida, Venezuela. Innovar, 27(64), 91-114. 10.15446/innovar. $\mathrm{v} 27 \mathrm{n} 64.62371$

Muñoz, M. (2017). Relaciones interpersonales y desempeño laboral del personal de enfermería de neonatología Hospital Arzobispo Loayza Lima 2015. (Tesis de maestria). Universidad César Vallejo.

Parra, Y. (2012). Comunicación interna en el mejoramiento de las relacioes interpersonales del personal administrativo de los Institutos Universitarios de Tecnología de Maracaibo. 10(2001), 111-119. Recuperado de http://ojs. urbe.edu/index.php/cicag/article/view/619

Robbins, S. (1999). Comportamiento Organizacional (Prentice H). México.

Robbins, Sterphen y Coulter, M. (2013). Administración (Pearson Ed). México.

Salazar, S. (2018). Relaciones Interpersonales y el Desempeño Laboral en la Oficina Registral del Reniec del Callao - 2017. (Tesis de maestria). Universidad César Vallejo.

San Martín, S. (2013). La confianza, la sastisfacción, las normas relacionales, el oportunismo y la dependencia como antecedentes del compromiso organizacional. Contaduria y Administración, 58(2), 11-38. Retrieved from http://www.cya.unam.mx/ index.php/cya/article/view/135/135

Sánchez, A., Martínez, R. y Moreno, J. (2017). Gestión por Competencias Laborales en el Contexto del Proceso de Cambios Políticos y Económicos en Cuba. Innovar, 27(66), 169184. 10.15446/innovar.v27n66.66811

Sanín, J. A. y Salanova, M. (2014). Satisfacción laboral: el camino entre el crecimiento psicológico y el desempeño laboral en empresas colombianas industriales y de servicios. Universitas Psychologica, 13(1), 1-22. 10.11144/Javeriana.UPSY13-1.slcp

Santos, G. (2016). Influencia de las relaciones interpersonales en el desempeño laboral de los trabajadores de la Municipalidad Provincial de Cajabamba - Agosto 2016. Universidad Privada del Norte.

Serrano, J. y Barba, M. I. (2012). La gestión de recursos humanos en las corporaciones locales. Cuadernos de Gestión, 12(2), 149168. $10.5295 / \mathrm{cdg} .100233 \mathrm{js}$

Tamayo, M. (1997). El Proceso de la Investigación cientifica (Limusa S.A). México.

Torres, E. y Zegarra, S. (2015). Clima organizacional y desempeño laboral en 
las instituciones educativas bolivarianas de la ciudad Puno 2014 - Perú.Comuni@ cción, 6(2), 5-14. Retrieved from http:// www.scielo.org.pe/scielo.php?script $=$ sci arttext\&pid=S2219-71682015000200001

Vargas, S. y Flores, M. (2019). Cultura organizacional y satisfacción laboral como predictores del desempeño laboral en bibliotecarios. Investigacion Bibliotecologica, 33(79), 149-176. 10.22201/ iibi.24488321xe.2019.79.57913

Vásquez, L. (2015). Relaciones interpersonales $y$ desempeño laboral en los trabajadores del Centro de Salud Morales, 2015. (Tesis de maestria). Universidad César Vallejo.
Willman, S. y Velasco, M. I. (2011). Relación en las percepciones del estilo de liderazgo del jefe inmediato con el desempeño laboral de los estudiantes en práctica de la Universidad ICESI. Estudios Gerenciales, 27(118), 67-84. Retrieved from https://www.sciencedirect. com/science/article/pii/S0123592311701462

Yañez, R., Arenas, M. y Ripoll, M. (2010). El impacto de las relaciones interpersonales en la satisfacción laboral general. Liberabit. Revista de Psicología, 16(2), 193-201. Retrieved from http://www.scielo.org.pe/pdf/ liber/v16n2/a09v16n2.pdf 